\title{
Associação entre midazolam e detomidina na medicação pré-anestésica para indução da anestesia geral com cetamina em potros
}

\author{
[A combination study of midazolam and detomidine in the premedication anesthesia for the induction of general \\ anesthesia with ketamine in foals] \\ J.A. Marques ${ }^{1}$, D.A. Pereira ${ }^{2}$, I.C.S. Marques ${ }^{3}$ \\ ${ }^{1}$ Faculdade de Ciências Agrárias e Veterinárias - UNESP \\ Via de Acesso Prof. Paulo Donato Castellane, s/n \\ 14884-900 - Jaboticabal, SP \\ ${ }^{2}$ Aluna de pós-graduação - FCAV-UNESP - Jaboticabal, SP \\ ${ }^{3}$ Aluna de graduação - Instituição Moura Lacerda - Ribeirão Preto, SP
}

\begin{abstract}
RESUMO
Empregou-se a associação midazolam e detomidina para indução de anestesia com cetamina em 16 potros, machos e fêmeas, entre três e seis meses de idade, distribuídos aleatória e equitativamente em dois grupos (GI e GII). A todos os animais foram administrados midazolam, via intramuscular, na dose de $0,2 \mathrm{mg} / \mathrm{kg}$, e após 15 minutos, detomidina, via intravenosa, na dose de $0,02 \mathrm{mg} / \mathrm{kg}$. Os animais do GII receberam cetamina pela via intravenosa, dose $2,0 \mathrm{mg} / \mathrm{kg}$, três minutos após a administração de detomidina. Quinze minutos após o midazolam, ocorreram sedação e ligeira ataxia, e dois minutos após a administração da detomidina, decúbito lateral em todos os potros, com miorrelaxamento e presença dos reflexos de deglutição e miorrelaxamento, anal e oculo-palpebral. A associação midazolam/detomidina e cetamina provocou ausência dos reflexos de deglutição. Para todos os animais, o tempo de recuperação foi de 45-60 minutos, e temperatura retal e frequência respiratória permaneceram estáveis. Ocorreram bradicardia, bloqueio atrioventricular de segundo grau e aumento das pressões arteriais sistólica, diastólica e média após dois minutos da administração da detomidina. A associação midazolam/detomidina e cetamina demonstrou ser um método eficiente e seguro para a anestesia de potros hígidos.
\end{abstract}

Palavras-chave: potro, cetamina, detomidina, midazolam, anestesia

\begin{abstract}
A combination of midazolam, $0.2 \mathrm{mg} / \mathrm{kg}$ body weight given via intramuscular, and detomidine, $0.02 \mathrm{mg} / \mathrm{kg}$ body weight given via intravenous (IV), was evaluated as a method for induction of anesthesia with ketamine, $2.0 \mathrm{mg} / \mathrm{kg}$ body weight given via IV in foals. Sixteen male and female foals aging from three to six-month old were distributed into two groups. Both groups were first injected with midazolam and with detomidine 15 minutes later. Three minutes later, ketamine was injected in the foals. Sedation and light ataxia were observed 15 minutes after midazolam administration. Bradycardia, atrioventricular block, increased blood pressure, lateral recumbency, and muscle relaxation were observed two minutes after detomidine. At that time, the oculopalpebral, anal, and cogh reflexes were present. The association of midazolam/detomidine and ketamine caused absence of the swallow reflexes. The recovery time was from 45 to 60 minutes for animals from GI and GII groups, respectively. Respiratory rate and body temperature remained unchanged. The combination of midazolam/detomidine and ketamine is a safe, good, and efficient method for anesthesia in healthy foals.
\end{abstract}

Keywords: foal, ketamine, detomidine, midazolam, anesthesia

Recebido em 23 de março de 2009

Aceito em 25 de novembro de 2009

Email: jmarques@fcav.unesp.br 


\section{INTRODUÇÃO}

O tamanho e o temperamento do equino fazem com que os procedimentos anestésicos sejam relativamente de maior risco para o animal e para o médico veterinário. A anestesia em equinos deve ser caracterizada por indução rápida e suave, requerer pequeno volume do agente indutor, produzir adequado relaxamento muscular, proporcionar analgesia, não alterar significativamente os parâmetros cardiopulmonares e, por fim, proporcionar uma recuperação livre de excitação (Fantoni et al., 1999).

Os diferentes procedimentos clínicos, radiográficos e intervenções cirúrgicas incluemse entre as principais indicações da medicação pré-anestésica em equinos. Até o presente, existe uma quantidade satisfatória de fármacos que são recomendados para uso na pré-anestesia, mas a maioria não reúne propriedades ideais como único agente. Atualmente, as associações de benzodiazepínicos e tranquilizantes são indicadas para procedimentos de anestesia em várias espécies animais, inclusive a equina. Essas associações visam potencializar os efeitos indesejáveis e reduzir os considerados indesejáveis e, ainda, diminuir a dose total da anestesia geral requerida (Dike, 1993).

Os benzodiazepínicos são associados com outros sedativos, analgésicos opioides ou não para promover uma sedação adicional e relaxamento muscular (Brock e Hildebrand, 1990). Os fármacos pertencentes ao grupo dos $\alpha$-2agonistas são atualmente utilizados com maior frequência em equinos como sedativos e/ou analgésicos (Muir e Hubbell, 1991). A detomidina, um dos agentes alfa-2-agonistas mais comumente utilizados na sedação e medicação pré-anestésica em equinos (Chambers et al., 1993), pode ser recomendada para intervenções cirúrgicas na posição quadrupedal.

Os avanços na área de anestesiologia veterinária e pesquisas com associações de fármacos têm sido objeto de frequentes investigações. Assim, o presente estudo teve os objetivos de avaliar os efeitos da associação midazolam e detomidina como pré-anestésicos para indução de anestesia com cetamina em potros, sua ação sobre as pressões arteriais sistólica, diastólica e média, temperatura retal e frequências cardíaca e respiratória, e seu efeito na sedação, hipnose e outras possíveis implicações clínicas.

\section{MATERIAL E MÉTODOS}

Utilizaram-se 16 potros, machos e fêmeas, considerados clinicamente sadios, de diversas raças, com idades entre três e seis meses e média de peso de $152 \mathrm{~kg}$. Os potros, distribuídos em dois grupos, foram pesados e tiveram mensuradas a temperatura retal (TR) e as frequências cardíaca (FC) e respiratória (FR). Também foram registradas as pressões arteriais sistólica (PAS), diastólica (PAD) e média (PAM). Esses parâmetros foram anotados $\mathrm{e}$ formaram a seu tempo os momentos: M0, M1, M2, M3, M4, M5, M6 e M7. No grupo I, foram formados M0, inicial ou basal; M1, correspondente a 15 minutos após a administração de midazolam $^{1}$; e M2, M3, M4, M5, M6 e M7, correspondentes a 2, 3, 15, 25, 35 e 45 minutos após a administração de detomidina $^{2}$, respectivamente. No grupo II, formaram-se M0, inicial ou basal; M1, correspondente a 15 minutos após administração de midazolam; M2, dois minutos após a administração de detomidina; M3, correspondente a três minutos após administração de detomidina e de cetamina; e M4, M5, M6 e M7, correspondentes a 10, 20, 30 e 40 minutos após a administração de cetamina ${ }^{3}$, respectivamente.

Nos potros em estação, contidos em tronco individual, procedeu-se à implantação de cânula de polietileno $\mathrm{n}^{\circ} 190^{4}$ no ramo transversal da artéria facial, que foi lavada com solução heparinizada ${ }^{5}$. As pressões arteriais sistólica, diastólica e média foram obtidas após a conexão da cânula a um transdutor de pressão ligado a um polígrafo $^{6}$. O aparelho foi calibrado com auxílio de manômetro de mercúrio, e os dados registrados em folha de papel milimetrado, formando uma escala de 0 a 250. Os valores de PAD e PAS foram registrados, respectivamente, nos limites inferior e superior da escala. A partir desses registros, foi calculada a pressão de pulso (PP), em que PP=PAS- PAD. A obtenção da

${ }^{1}$ Dormonid $15 \mathrm{mg} / 3 \mathrm{~mL}$ - Roche, São Paulo, Brasil.

${ }^{2}$ Domosedan - Ciba Geigy - São Paulo, Brasil.

${ }^{3}$ Vetanarcol - König do Brasil Ltda. - São Paulo, Brasil.

${ }^{4} \mathrm{CPL}$ - Medical Produtos Médicos Ltda - São Paulo, Brasil.

${ }^{5}$ Liquemine - Roche, São Paulo, Brasil.

${ }^{6}$ Narco Biosystems - DescModelDMP-4B - Texas, EUA. 
pressão média foi obtida pela equação proposta por STURKIE (1970), em que PAM= PAD+ 3/8PP.

Os exames eletrocardiográficos ${ }^{7}$ foram obtidos empregando-se a derivação baseápice, com a fixação dos eletrodos nos locais correspondentes. Todos os animais receberam midazolam, por via intramuscular, e detomidina, via intravenosa, respectivamente, nas doses de 0,2 e $0,02 \mathrm{mg} / \mathrm{kg}$ de peso corpóreo.

A sequência experimental ocorreu da seguinte forma: no grupo I, os potros receberam midazolam, aguardaram 15 minutos (M1) e receberam detomidina, completada com solução fisiológica, devido à pequena quantidade, até o volume total da seringa. A partir de M3 e a intervalos de 10 minutos, novas mensurações foram realizadas para obter M4, M5, M6 e M7. No grupo II, após receberem a mesma prémedicação do grupo I, e decorridos três minutos da administração de detomidina (M3), administrou-se cetamina, na dose de $2,0 \mathrm{mg} / \mathrm{kg}$, a intervalos de 10 minutos, para obter M4, M5, M6 e M7.

Todos os animais foram avaliados, para observação de possíveis alterações comportamentais. O delineamento experimental empregado foi o de parcelas, grupos I e II e subparcelas, M0 a M7. A comparação das médias foi feita pelo teste Tuckey, considerandose o nível de probabilidade de $5 \%$ e $1 \%$.

\section{RESULTADOS E DISCUSSÃO}

Foram observados sinais de sedação após a administração de midazolam. Esse fato é explicado pela ação dos benzodiazepínicos, que reduzem a atividade funcional do hipotálamo e córtex, com ação sobre o GABA, inibindo o sistema nervoso central e promovendo ação tranquilizante, hipnótica, ansiolítica e miorrelaxante (Spinosa et al., 2002). Dessa forma, foram evidentes os sinais de sedação 15 minutos após o emprego do midazolam. Assim, ambos os fármacos agonistas alfa-2 e benzodiazepínicos exerceram efeitos sedativos e ansiolíticos, quando administrados como prétratamento. McKenzie et al. (2004) descreveram que o midazolam é comumente usado como o

${ }^{7}$ RFY6NEK Seschskanal Eletrokardiograf- DDR. sedativo de escolha na unidade de terapia intensiva e que, de acordo com Hellyer et al. (1991) e Ilkiw et al. (1998), a administração conjunta de cetamina e benzodiazepínico representa uma das associações mais empregadas para anestesia e pré-medicação em pequenos animais, por proporcionar segurança e efeitos depressores mínimos, além de rápida recuperação. Apesar de o midazolam frequentemente produzir alterações de comportamento, tais como excitação, vocalização e agressividade na contenção de gatos, esse fármaco demonstra maior potência do que o diazepam, o agente desse grupo que é mais frequentemente empregado em animais.

Neste estudo, ficou demonstrado que o midazolam isolado ou associado à cetamina e/ou detomidina apresentou efeitos benéficos, já observados em outras espécies (Hellyer et al., 1991; Jacobson e Hartsfield, 1993; Lesser et al., 1993; Guedes et al., 2002). A ataxia, o abaixamento da cabeça e a indiferença ao ambiente conferida pelo midazolam facilitaram a abordagem e a contenção física dos potros, uma vez que estes se apresentavam sedados, demonstrando que essa substância na dose preconizada de $0,2 \mathrm{mg} / \mathrm{kg}$ induziu os efeitos hipnóticos e miorrelaxantes (Fantoni e Cortopassi, 2002; Massone, 2003).

O decúbito lateral e o miorrelaxamento, evidenciados após a aplicação da detomidina, provavelmente ocorreram pelo fato de os fármacos agonistas alfa-2-adrenérgicos poderem estar associados a derivados fenotiazínicos para a obtenção de efeito sedativo intenso que ocorre em equinos e caninos. Também ocorreram tais efeitos quando estavam associados a opioides para a produção de neuroleptoanalgesia em equinos ou a anestésicos dissociativos, como a cetamina, para a sedação de felinos e caninos agressivos (Natalini, 2007). Brock e Hildebrand (1990) sugeriram que a inclusão do diazepam no protocolo de indução anestésica por éter glicerilguaiacol e cetamina pode reduzir o consumo de anestésico inalatório. Uma possível explicação para esse fato é o relaxamento muscular e o efeito hipnótico adicional produzido pelo diazepam.

Particularmente neste estudo, o grau intenso de ataxia descrito para a detomidina não foi observado, uma vez que a associação midazolam 
e detomidina provocou decúbito lateral, evidenciando relaxamento muscular e hipnose ideais para procedimentos anestésicos em potros.

Os anestésicos inalatórios voláteis causam depressão cardiovascular dose dependente, seja por depressão direta do miocárdio ou por diminuição da atividade simpática (Steffey, 1996). Quando se deseja manutenção da estabilidade cardiovascular, uma alternativa aos anestésicos voláteis é o uso de técnicas de anestesia injetável balanceada. O emprego adequado dos benzodiazepínicos facilita os procedimentos de anestesia. De acordo com Brondani et al. (2003), em cães observou-se que o uso da cetamina provoca aumento da frequência cardíaca devido provavelmente à ação simpatomimética desse fármaco, efeito este que provavelmente foi minimizado pelo uso concomitante de midazolan e de óxido nitroso, evidenciando que a associação cetaminamidazolam não provocou depressão da função cardiovascular. Observações semelhantes também foram relatadas por Hellyer et al. (1991), Jacobson e Hartsfield (1993) e por Lesser et al. (1993), quando o óxido nitroso foi adicionado à cetamina-diazepam em suínos. Portanto, os resultados obtidos neste estudo confirmam que a estabilidade cardio-respiratória conferida após o uso do midazolam, pela via intramuscular, permite indicá-lo como opção segura para uso em procedimentos de préanestesia de potros hígidos.

Natalini (2007) relatou que o efeito dos agonistas é a inibição da liberação de norepinefrina e o bloqueio dos receptores noradrenérgicos présinápticos, levando à sedação, analgesia, relaxamento muscular, inibição da liberação de insulina e consequente hiperglicemia, diurese osmótica, depressão respiratória em doses elevadas e êmese por ativação do centro do vômito. De acordo com Short (1992), a diurese observada após o uso da detomidina é devido provavelmente à ação desse fármaco no sistema renal, o qual age diminuindo a secreção do hormônio antidiurético. Segundo Kallio et al. (1988), em pôneis, a detomidina provavelmente foi a responsável pela diminuição na atividade da glândula pituitária-adrenocortical, e a diurese foi provavelmente causada pela supressão de vasopressina e da secreção de insulina pela detomidina (Taylor et al., 1992).
Neste estudo, não foi observado aumento na frequência de micções, provavelmente devido à dose de detomidina empregada. Estudos prévios já demonstraram o efeito diurético da detomidina em cavalos acordados e anestesiados com halotano (Steffey et al., 2000).

Trabalhos verificaram que os fármacos alfa-2agonistas causam bradicardia, comumente acompanhada por bloqueio atrioventricular (Hodgson e Dunlop, 1990; England e Clarke, 1996), bloqueio sinoatrial menos frequente e redução no débito cardíaco, e, na maioria dos animais os bloqueios são mais intensos nos primeiros minutos após a administração. Após esse período, a frequência cardíaca eleva-se gradativamente e os bloqueios desaparecem. A bradicardia e os bloqueios cardíacos persistem por mais tempo após administração de romifidina do que com xilazina ou detomidina (England e Clarke, 1996). Segundo esses autores, em equinos saudáveis, a administração intravenosa de xilazina, detomidina, medetomidina ou romifidina produz hipertensão inicial transitória dose dependente, seguida por prolongada redução na pressão arterial, mesmo com o uso de pequenas doses.

Portanto, neste estudo, decorridos dois a cinco minutos da administração da detomidina pela via intravenosa, houve evidente queda da frequência cardíaca e não foi observada ocorrência de bloqueio atrioventricular de segundo grau em nenhum animal, 15 minutos após a administração de midazolam (M1), demonstrando que esse fármaco, administrado pela via intramuscular em potros, não interfere na frequência ou no ritmo cardíaco. Contudo, observou-se que a administração de detomidina induziu bloqueio atrioventricular de segundo grau e episódios de bradicardia aos dois minutos, que se estabilizaram rapidamente. A menor dose de detomidina pode ter contribuído para que o tempo de decorrência desses efeitos indesejáveis fosse mais curto, pois parece ter ocorrido efeito sinérgico benéfico para indução de anestesia em potros entre o midazolan e a detomidina.

A elevação dos valores de PAS, PAD e PAM demonstrou que esse fármaco provocou hipertensão transitória, entre dois e cinco minutos da administração intravenosa, e houve, após esse período, progressivo retorno aos valores basais (Fig. 1, 2 e 3). Resultados 
semelhantes foram descritos por Fantoni et al. (1999), que relataram aumento significativo da pressão arterial em equinos, após 15 minutos da administração de agentes alfa-2-agonistas, particularmente a detomidina. Efeito semelhante foi observado por Wagner et al. (1991), ao atribuírem a hipertensão à estimulação alfa-adrenérgica pós-sináptica periférica, que resultou em vasoconstrição e aumento da pressão arterial. Também foi semelhante ao efeito observado por Muir e Mason (1993) em relação à xilazina e à detomidina.

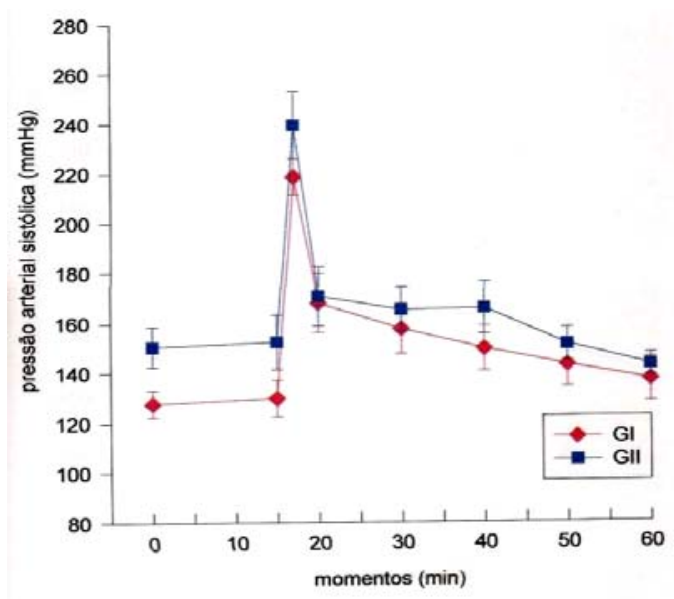

Figura 1. Valores de pressão arterial sistólica (mmHg) obtidas em potros tratados com midazolam e detomidina (GI) ou midazolam, detomidina e cetamina (GII).

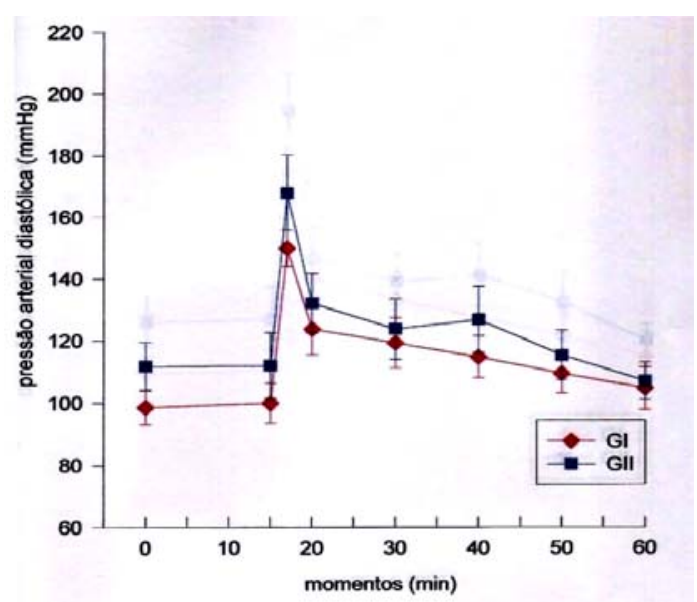

Figura 2. Valores de pressão arterial diastólica (mmHg) obtidas em potros tratados com midazolam e detomidina (GI) ou midazolam, detomidina e cetamina (GII).

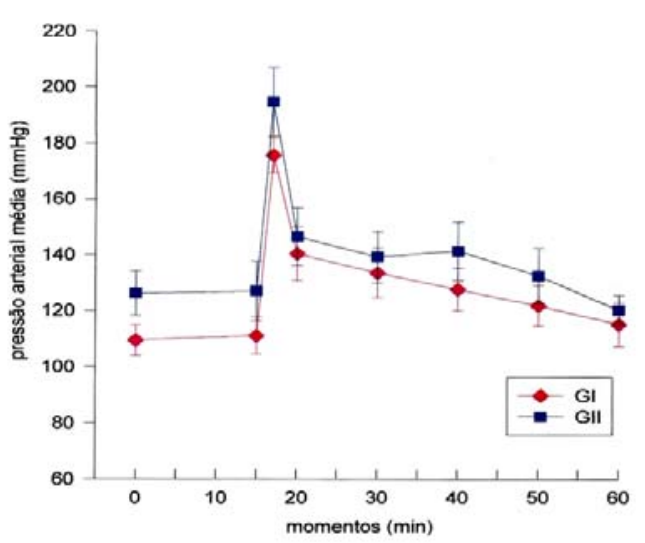

Figura 3. Valores de pressão arterial média (mmHg) obtidas em potros tratados com midazolam e detomidina (GI) ou midazolam, detomidina e cetamina (GII).

A frequência respiratória permaneceu inalterada durante toda a observação, sugerindo estabilidade ventilatória. Em equinos, Luna et al. (1990) verificaram diminuição da frequência respiratória com o uso de xilazina ou romifidina, e esse efeito indesejável foi mais acentuado com a romifidina.

Os resultados desta pesquisa demonstraram que o uso da detomidina em potros provocou efeitos bradicárdicos e de bloqueio atrioventricular, subsequentes à hipertensão arterial. Eles são mais evidentes, segundo England et al. (1992), quando a detomidina é administrada em bolus, e ainda podem ser verificados efeitos indesejáveis de ataxia e recuperações prolongadas. Quanto ao sistema cardiovascular, Daunt et al. (1993) relataram que o uso da detomidina resultou em diminuição do índice cardíaco em um terço.

Os anestésicos dissociativos, como cetamina e tiletamina, são utilizados para a obtenção de sedação em animais agressivos. É obrigatório o uso associado a outros fármacos tranquilizantes e relaxantes musculares, pois os derivados fenciclidínicos produzem intensa rigidez muscular, catatonia, excitação, hipertensão arterial e taquicardia, além de aumentarem a produção de secreções salivares e brônquicas (Natalini, 2007). Selmi et al. (2005) descreveram que o aumento transitório da frequência cardíaca, logo após a administração de cetaminamidazolam, decorre da ação simpatomimética do agente dissociativo. Relatos semelhantes foram citados por Cullen e Reynoldson (1997) em cães 
pré-medicados com tiletamina-zolazepam e anestesiados com propofol.

A cetamina, quando utilizada como único agente indutor, provoca em equinos efeito cataleptoide de forma evidente. Para inibir esses efeitos, Massone (2003) recomenda o uso de fármacos como as benzodiazepinas, que, devido à sua ação no sistema nervoso central, provocam miorrelaxamento, hipnose e ausência de excitação. $\mathrm{O}$ que pode ser comprovado neste estudo é que o midazolam e a detomidina inibiram os efeitos indesejáveis citados para a cetamina, e, como tal, podem ser recomendados para procedimentos como as cirurgias de curta duração ou, ainda, indução de anestesia geral (Hellyer et al., 1991, Steffey, 1996). Com base nos resultados obtidos neste experimento, podese indicar, com certo grau de segurança, o emprego da associação midazolam/detomidina e/ou cetamina para diversos procedimentos que demandam contenção química em potros hígidos, tendo em vista a realização de intubação orotraqueal de forma suave, pois essa associação é capaz de inibir os reflexos laringotraqueais.

A cetamina, um anestésico dissociativo, possui ação simpatomimética e estimula a FC, resultando no aumento do débito cardíaco. Exerce vasoconstrição periférica, que eleva consideravelmente a pressão arterial, entretanto não altera a pressão venosa. É indicada em casos de choques, especialmente o hemorrágico. Neste trabalho, observou-se taquicardia e hipertensão, assim, verificou-se que a hipertensão ocorreu devido à ação da detomidina, embora por curto período, e manteve elevadas as pressões arteriais sistólica, diastólica e média. A frequência cardíaca nos dois grupos experimentais diminuiu significativamente dois minutos após a administração de detomidina, e permaneceu por 30 minutos nos animais do grupo I e 10 minutos nos do grupo II. Assim, a cetamina foi capaz de melhorar a frequência dos batimentos cardíacos, demonstrando que esse fármaco provoca efeitos taquicardizantes em potros, sendo essa característica importante para procedimentos de anestesia em animais com bradicardia.

Observou-se, ainda, que todos os potros apresentaram evidente grau de relaxamento muscular, pois permitiram manipulações incruentas e colocação de máscaras que são empregadas em procedimentos de anestesia, e nos animais do grupo II, intubação orotraqueal. A recuperação anestésica foi destituída de efeitos colaterais, e os potros recuperaram a posição de estação quadrupedal, sem evidências de eventos excitatórios e espasmos musculares.

\section{CONCLUSÕES}

Em potros, o midazolam não interferiu nos parâmetros cardiorrespiratórios e provocou efeito ansiolítico 15 minutos após a administração intramuscular; a detomidina provocou bloqueio atrioventricular de segundo grau, bradicardia e hipertensão arterial aos dois minutos após a administração intravenosa; a associação midazolam/detomidina inibiu os efeitos colaterais produzidos pela cetamina, e foi observado miorrelaxamento capaz de provocar o decúbito lateral; a associação midazolam/ detomidina e/ou midazolam/detomidina/cetamina não alterou os parâmetros respiratórios e a temperatura retal; a associação midazolam/ detomidina/cetamina demonstrou ser segura e prática, portanto seu uso pode ser recomendado como agente indutor para potros em diferentes procedimentos anestesiológicos.

\section{REFERÊNCIAS BIBLIOGRÁFICAS}

BRONDANI, J.T.; NATALINI, C.C.; PIPPI, N.L. et al. Ketamine, midazolam and nitrous oxide anesthesia in dogs submitted to cervical esophagoplasty. Cienc. Rural, v.33, p.1075-1080, 2003.

BROCK, N.; HILDEBRAND, S.V.A. Comparison of xylazine-diazepam-ketamine and xylazineguaifenesin-quetamine in equine anesthesia. Vet. Surg., v.9, p.468-474, 1990.

CHAMBERS, J.P.; LIVINGSTON, A.; WATERMAN, A.E. et al. Analgesic effects of detomidine in thoroughbred horses with chronic tendon injury. Res. Vet. Sci., v.54, p. 52-56, 1993.

CULLEN, L.K.; REYNOLDSON, J.A. Effects of tiletamine-zolazepam premedication on propofol anaesthesia in dogs. Vet. Rec., v.140, p.363-366, 1997.

DAUNT, D.A.; DUNLOP, C.I.; CHAPMAN, P.L. et al. Cardiopulmonary and behavioral responses to computer-driven infusion of detomidine in standing horses. Am. J. Vet. Res., v.54, p.2075-2082, 1993.

DIKE, T.M. Sedatives, tranquilizers, and stimulants. Vet. Clin. N. Am.: Equine Pract., v.9 p.621-631, 1993. 
ENGLAND, G.C.W.; CLARKE, K.W.; GOOSENS, L. A comparison of the sedative effects of three a2-adrenoceptor agonists (romifidine, detomidine and xylazine) in the horse. J. Vet. Pharm. Ther., v.15, 194-201, 1992.

ENGLAND, G.C.W.; CLARKE, K.W. Alpha2adrenoreceptor agonists in the horse - a review. $\mathrm{Br}$. Vet. J., v.152, 641-657, 1996.

FANTONI, D.T.; CORTOPASSI, S.R.G. Anestesia em cães e gatos. São Paulo: Roca, 2002. 389p.

FANTONI, D.T.; FUTEMA, F.; CORTOPASSI, S.R.G. et al. Comparative evaluation of acepromazine, detomidine and romifidine in horses. Cienc. Rural, v.29, p.45-50, 1999.

GUEDES, A.G.P.; NATALINI, C.C. Anesthesia in horses with colic syndrome - analysis of 48 cases and literature review. Cienc. Rural, v.32, p.535$542,2002$.

HELLYER, P.W.; FREEMAN, L.C.; HUBBELL, J.A.E. Induction of anesthesia with diazepamketamine and midazolam-ketamine in greyhounds. Vet. Surg., v.20, p.143-147, 1991.

HODGSON, D.S.; DUNLOP, C.I. General anesthesia for horses with specific problems. Vet. Clin. N. Am.: Equine Pract., v.6, p.625-650, 1990.

ILKIW, J.E.; SUTER, C.; McNEAL, D. et al. The optimal intravenous dose of midazolam after intravenous ketamine in healthy cats. J. Vet. Pharm. Ther., v.21, p.54-61, 1998.

JACOBSON, J.D.; HARTSFIELD, S.M. Cardiovascular effects of intravenous bolus administration and infusion of ketamine-midazolam in dogs. Am. J. Vet. Res., v.54, p.1710-1714, 1993.

KALLIO, A.; KOULU, M.; SCHEININ, H. et al. Acute effects of medetomidine, a selective alpha-2 adrenoceptor agonist, on anterior pituitary hormone and cortisol secretion in man. Acta. Endocrinol., v.119, p.11-15, 1988.

LESSER, T.; EBNER, E.; ZWIENER, U. Ketamine diazepam $\mathrm{N} 2 \mathrm{O}$ combination anesthesia a new circulatory system-sparing type of anesthetic in experimental surgery. Res. Exp. Med., v.193, p.207$211,1993$.

LUNA, S.P.L.; VIEIRA, F.A.F.; PAVANI, J. et al. Comparação entre detomidina e romifidina em equinos. Hora Vet., v.89, p.56-60. 1990.

MASSONE, F. (Ed). Anestesiologia veterinária: farmacologia e técnicas. 4.ed. Rio de Janeiro: Guanabara Koogan, 2003. 326p.
McKENZIE, C.A.; McKINNON, W.; NAUGHTON, D.P. et al. Differentiating midazolam over-sedation from neurological damage in intensive care unit. Crit. Care, v.9, p.R32-R36, 2005.

MUIR, W.W.; HUBBELL, J.A.E. Equine Anesthesia. St Louis: Mosby-Year Book, 1991. p.247-280.

MUIR, W.W.; MASON, D.E. Effects of diazepam, acepromazine, detomidine, and xylazine on thiamilal anesthesia in horses. J. Am. Vet. Med. Assoc., v.203, p.1031-1038, 1993.

NATALINI, C.C. Teorias e técnicas em anestesiologia veterinária. Porto Alegre: ArtMed, 2007. p.53-56.

SELMI, A.L.; FIGUEIREDO, J.P.; MENDES, A.M. et al. Infusão contínua de propofol em gatos pré-medicados com cetamina-midazolam. Arq. Bras. Med. Vet. Zootec., v.57, p.295-299, 2005.

SHORT, C.E. Alpha2 - agents in animals: sedation, analgesia and anaesthesia. Vet. Pract. Publ. Comp., v.1, p.85, 1992.

SPINOSA, H.L.; GÓRNIAK, S.L.; BERNARDI, M.M. Farmacologia aplicada à medicina veterinária. 3.ed. Rio de Janeiro: Guanabara Koogan, 2002. 752p.

STEFFEY, E.P. Inhalation anesthetics. In: LUMB, W.V.; JONES, E.W. (Eds). Veterinary anesthesia. 3.ed. Baltimore: Williams \& Wilkins, 1996. p.297329.

STEFFEY, E.P., PASCOE, P.J., WOLINER, M.J et al. Effects of xylazine hydrochloride during isoflurane-induced anesthesia in horses. Am. J. Vet. Res., v.61, p.1225-1231, 2000.

STURKIE, P.D. Circulation in aves. Fed. Proc., v.29, p.1674-1679, 1970.

TAYLOR, P.M.; LUNA, S.P.L.; BREARLEY, J.C. et al. Physiological effects of total intravenous surgical anaesthesia using detomidineguaiphenesin-ketamine in horses. J. Vet. Anaesth., v.19, p.24-31, 1992.

WAGNER, A.E.; MUIR, W.W.; HINCHCLIFF, K.W. Cardiovascular effects of xylazine and detomidine in horses. Am. J. Vet. Res., v.52, p.651$657,1991$. 